\title{
Farms Monitoring System Based on Wireless Sensor Network
}

\author{
Wang Yong-jie, Guo Qiang
}

(Guilin university of Technology ,college of Information and Engineering, Guilin,china, 541004)

Email: wyongjie12@163.com

Key words: Wireless sensor network, CC2430, monitoring system

\begin{abstract}
This paper introduce the application of WSN in farms monitoring system, which proposed the realization method of monitoring based on CC2430.Discussed in detail the principle and structure of network,hardware circuit ,program flow chart,etc. The monitoring system is simple,small power consumption, wiring convenience,high safety.
\end{abstract}

\section{Introduction}

Farming is the production department, It convetred plant energy into animals energy through artificial breeding that have been humans domesticated animals , feeding to obtain meat, eggs, milk, wool and other animal products.Farming is the main part of agriculture,and the planting industry and listed as the two pillars of agricultural production.Farming is the extremely important link of material exchange between human and nature. Do a good job in farming is of great significance in the aspects of breeding production , promote the economic development, improvement of people's life, increase exports, strengthen national unity

One of the important conditions to develop the breeding, is suitable natural conditions. From scientific farming, breeding management level, realize modern farming, intelligent monitoring is an inevitable trend in the modern production development.This paper introduce the wireless sensor network monitoring system of farms, can be achieved onmonitoring parameters such as temperature, humidity, gas concentration and light,etc, and then achieve ideal environmental conditions, through manual adjustments to create a comfortable, healthy growth and living environment for animal.These can achieve better economic benefit and efficiency.

\section{The hardware design}

Wireless sensor network (WSN) is composed of a large number of micro sensor nodes deployed in monitoring area, through wireless communication mode to form a multiple hops self-organizing network system, its purpose is collaboration to perceive, collection and processing perception object information in the network coverage area,then sent to the observer. Wireless sensor network technology in the United States business week and technology to predict the future development of MIT technology review report, respectively is listed as one of the most influential in the 21st century 21 items of technology and one of the top 10 technology to change the world.

With the development of science and technology and the needs of the production, automatic monitoring technology has been widely applied in many fields. The current development trend of automatic monitoring instrument is a highly integrated, digital, generalization, automation, and intelligence. Do not need artificial participation during the operation of automatic monitoring instrument, reduces the operating costs, improve the reliability.

At present, the wireless sensor network (WSN) is playing an increasingly important role in environmental monitoring. Compared with the traditional means of environmental monitoring, environment monitoring system based on wireless sensor network (WSN) can work in harsh outdoor environment for a long time without specialist field of duty, at the same time with a large amount of data acquisition and monitoring the advantages of high precision, easy to deploy.

Sensor node is the basic component of wireless sensor network (WSN) units. It is composed of sensor module, the processor module, wireless communication module and power supply module. 
Since sensor nodes are usually the miniature of the embedded system, its processing power, storage capacity and communication ability are relatively weak, and through carrying energy limited battery power supply. According to the characteristics of sensor nodes, we design a used for monitoring environmental parameters in farms with chip CC2430 as the core of wireless sensor network node.

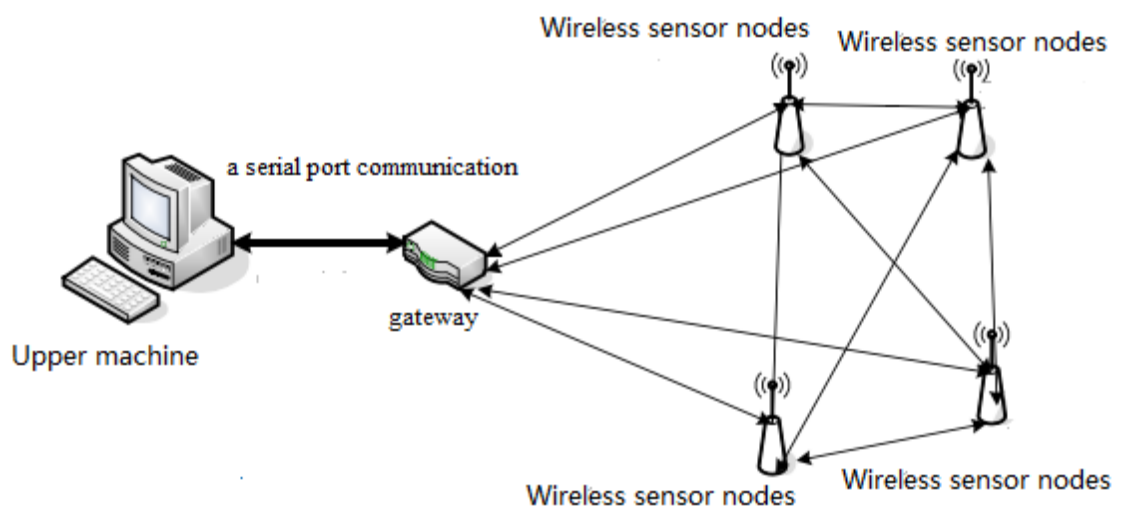

Fig1. The chart of whole hardware system

Based on the Zigbee standard product CC2430 is a real system chip (SoC) solution for CMOS. This solution can improve performance and meet ZigBee based 2.4 GHz ISM band application, and of low cost, low power requirements. It combines a high performance $2.4 \mathrm{GHz}$ DSSS (direct sequence spread spectrum) rf transceiver 8051 controller core and an industrial-strength compact and efficient. CC2430 design is a combination of 8 kbyte of RAM and powerful peripheral modules, and there are three different versions, they are based on different flash memory space of 32, 64, and 128 kbyte to optimize the complexity and cost of combination.

The whole system hardware structure diagram as shown in figure 1[1].Upper machine is interaction platform in users, network management and aintenance personnel with the wireless sensor network, users can through the PC software for wireless sensor network configuration and data information interaction with terminal equipment. PC using common computer.

\section{The architecture of wireless sensor network node}

The nodes of wireless sensor network usually consists of sensor module, processor module, wireless communication module and power module. Processor module and wireless communication module uses CC2430 chips, greatly simplifies the design of rf circuit.Sensors are mainly Temperature and humidity, light intensity sensor and gas concentration sensor.

This system is consisted of distributed sensor nodes, the receiving and sending of the Internet and user interface.The system block diagram is shown in figure 2.Sensor node is the basic unit of the network, the random distribution sensor nodes measured signals in the detection area, to deel whith primary treatment sensory data using the built-in processor.Due to the limitation of node power, the transmission distance is limited, so the node will be looking for adjacent nodes as transmission trunking, it sends the related information to the gateway node (Sink) through multiple hops in the secondary. The gateway node Is a special node, have relatively strong processor and large storage space, has more processing power and range.

The sensor nodes can be targeted arrangement in advance, thus has the relatively fixed network topology. Farms environment shows some characteristics, using different types of sensor is sensitive to perception, and generate the corresponding sensor output signal. From the angle of signal processing, sensor output signal can be approximately regarded as a non-stationary random process, using the method of signal detection and processing and combining with the characteristics of signals can be effectively detected signal environment parameters. 


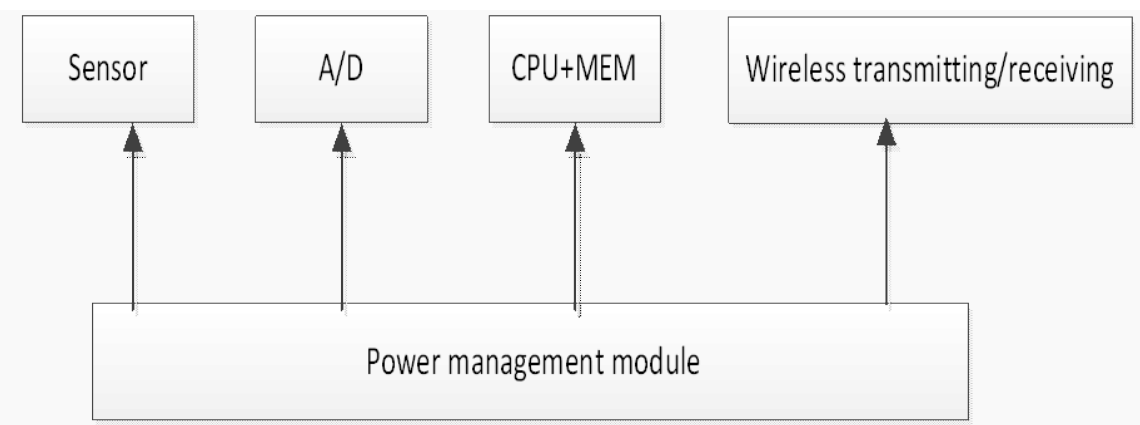

Figure 2. Wireless sensor nodes architecture

System adopts the bus structure, blocks organized, a multiple wireless temperature humidity monitoring area can be placed degree of acquisition node, network is convenient. About liquid crystal display, the use of a 3.5 -inch LCD display, can easily read each patch value of temperature and humidity,gas,etc.All kinds of data and information be clear at a glance. And it can set the alarm on the lower limit, and provide the combination of sound and light alarm and SMS alarm mode. When an exception occurs, lights flashing, short buzzer rang and warning messages. Flexible way of remote data transmission, can choose RS232/485, ethernet or GPRS communication methods such as sending data to the monitoring center database. Control center computer software is a powerful data processing function, can realize the monitoring area within the network real-time monitoring and data record, and query historical data, the expert data, history curve, etc. It can onnecte to the wide area network using any computer,that can be convenient to query data record.

\section{The design of the hardware circuit}

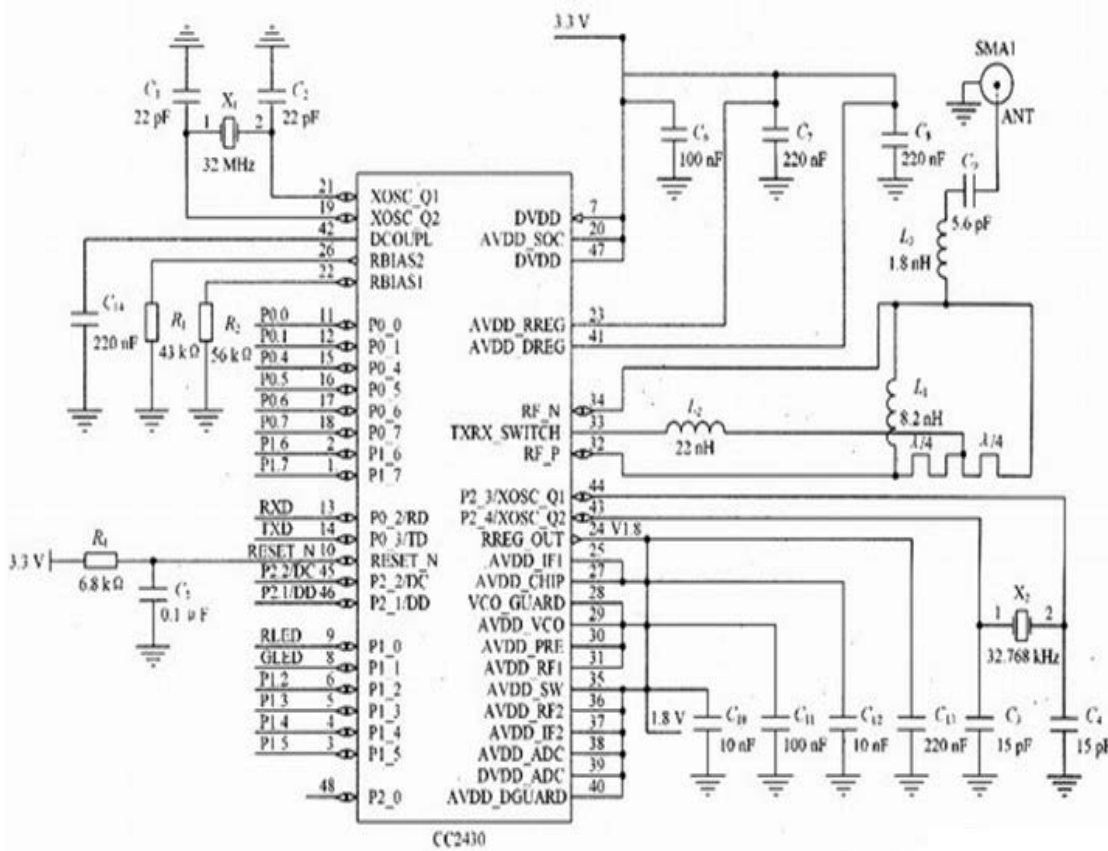

Figure 3. CC2430 basic circuit

The sensor nodes can be targeted arrangement in advance, thus has the relatively fixed network topology. Farm environment shows some characteristics, using different types of sensor is sensitive to perception, and generate the corresponding sensor output signal. From the angle of signal processing, sensor output signal can be approximately regarded as a non-stationary random process, using the method of signal detection and processing and combining with the characteristics of signals can be effectively detected signal environment parameters.CC2430 is a system on a chip, is used to implement embedded ZigBee application, introduced by chipcon company. CC2430 can run only need a few external components, its internal has integrated a lot of necessary circuit, so the less peripheral circuit can realize the function of signal to send and receive. Figure 3 for CC2430 basic 
circuit design[7].

In figure $3, C_{1}$ and $C_{2}$ are $22 p F$ capacitor, they connection $32 M H_{Z}$ crystal resonance circuit,the quartz crystals is used for normal work frequency. ${ }^{C_{3}}$ and $C_{4}$ are $15 p F$ capacitor, they connection $32.768 \mathrm{KH}_{Z}$ crystal resonance circuit,the quartz crystals is used for sleep work,thus reducing power consumption. $C_{5}=0.1 \mu \mathrm{F}$, used to remove some clutter interference, prevent SCM error reduction. $C_{6} \sim C_{8}$ is respectively $100 n F, 220 n F, 220 n F$, used as a filter, removing clutter interference makes more stable voltage. $C_{9}=5.6 \mathrm{pF}$, china-africa balance transformer is composed by ${ }^{C_{9}}$ and inductance $L_{1}, L_{2}, L_{3}$ and a PCB of microwave transmission line, the entire structure meet to ther equirements of RF input/output matching resistance ( $50 \Omega$ ). $L_{1}, L_{2}, L_{3}$ is respectively $8.2 n H, 22 n H, 1.8 n H, C_{10}, C_{11}, C_{12}$, and $C_{13}, C_{14}$ are capacitance without coupling , for the power filter, in order to improve the stability of the chip work.Bias resistors $R_{1}$ and $R_{2}$ is respectively $43 k \Omega, 56 k \Omega, R_{1}$ is used to set precision bias current for $32 M H_{z}$ crystal oscillator.

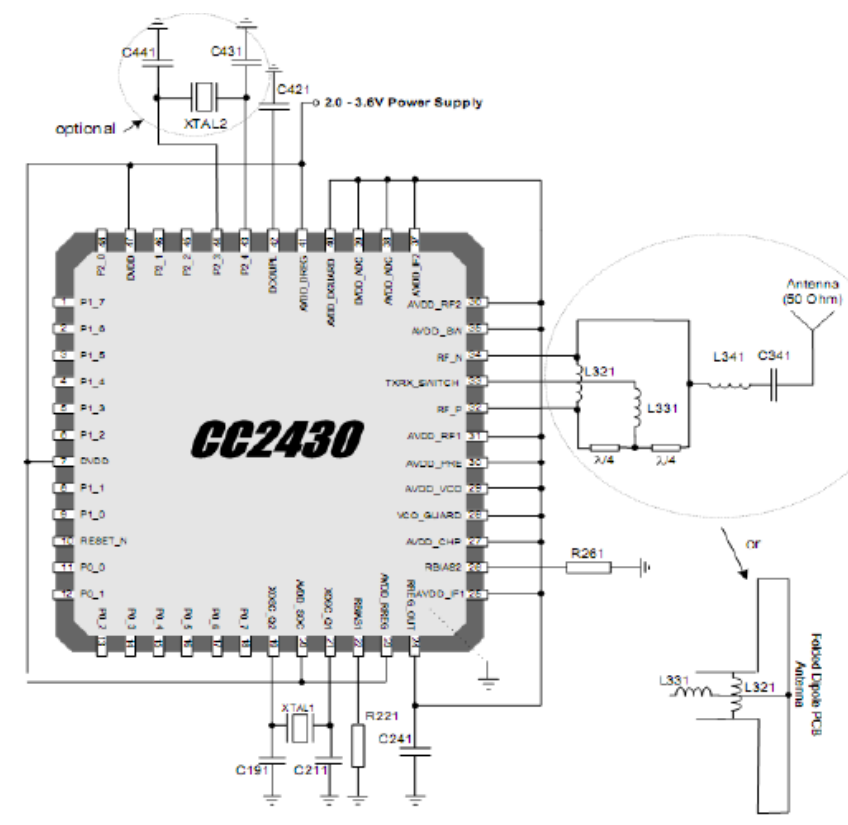

Figure 4. CC2430 Application circuit

CC2430 uses standard 8051 instruction set, with eight times the performance of the standard 8051 kernel. This is because its per clock cycle is a machine cycle, while in the standard 8051 it is 12 clock cycle as a machine cycle, get rid of the bus state way is wasted. Most of the execution time is a single instruction system clock cycles. Using fewer peripheral circuits that send and receive signals function, figure 4 is CC2430 typical application circuit, provides two antenna application circuit, unbalanced antenna (such as monopole antenna) and differential antenna (folded dipole antenna PCB)[4].

\section{The software design}

Software part needs to solve the problems include: the temperature and humidity, illumination and gas signal acquisition, Zigbee protocol stack, Zigbee communication, etc. Temperature and humidity, illumination and gas signal collection can be performed by internal CC2430 MCU. Sensor node application flow chart shown in figure 5 [6]. 


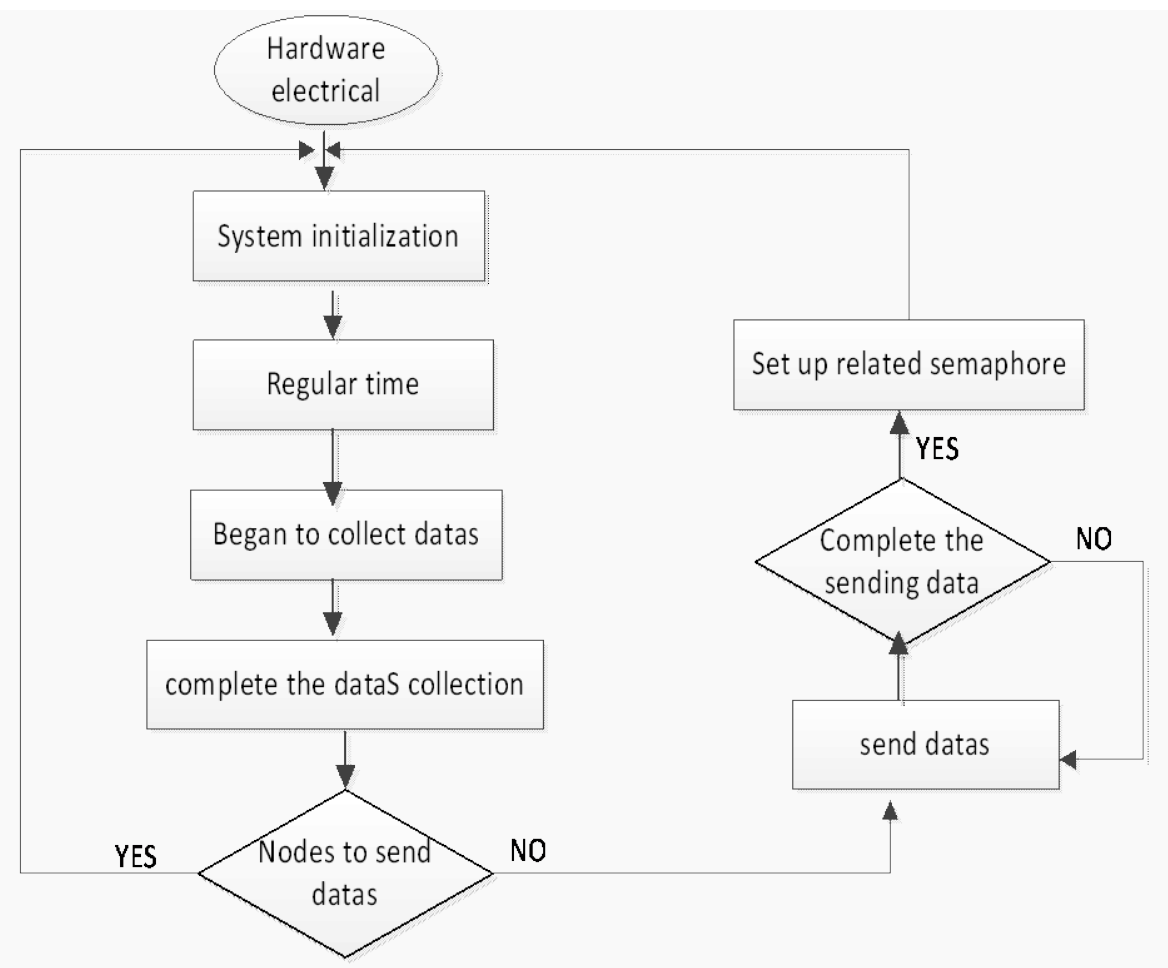

Figure 5. nodes application flow chart

When open the node power supply ,then system initialization and enter a dormant state until timing time arrive to be activated. Then the node control sensors for data acquisition. When all the sensor acquisition work finished packaged all the data sent to the base station. If the node is sending data, wait until after node sends the data to send the packet. On the other hand, is to send the data. When finished sending packets, return a semaphore it has completed the amount of data sent successfully,then enter a dormant state. Because the sampling frequency is same,the offset is maked according to the different node number, and the offset won't each data sent to the station at the same time, won't happen data conflicts.

Farm monitoring system, monitoring data and control commands were transferred between wireless sensor nodes, wireless routing nodes,wireless gateway and the monitoring center.Sensor nodes open the power supply, after the initialization, establishing the link to enter a dormant state.The interrupt be triggered when the wireless gateway receiving interrupt request,through routing nodes activating sensor nodes,send or receive data packets,continue to enter a dormant state after processed, waiting for another request activation.In the same channel only two nodes can communicate, by competition mechanism to obtain channel.Each node periodically sleep and listening to the channel.If the channel is idle ,the node will preempt channel actively.If the channel is busy, the node will retreat for a period of time according to retreat algorithm,then listening to the channel state again.In programming,mainly adopt the method of interrupt to complete the information receiving and sending.

The monitoring software tranform information using base station and the whole sensor network, the whole network node data accessed by the base station, realize the node collection of environmental information display, storage, query and network topology, and other functions,through the base station send commands to nodes in the network, in order to realize the control and management of network.

\section{Conclusions}

This paper introduces a set of farms monitoring system based on wireless sensor network (WSN), the system need not human intervention at run time, with the function of real-time monitoring, alarm, and query historical data. Farms monitoring system based on a lot of research work done on the basis of the data acquisition, display and remote transmission, etc.This system is based on 
intelligent purpose, animal husbandry and farming, it can provide efficient and practical environmental monitoring for you.

Farm monitoring and control system based on wireless sensor network,simple structure, small power consumption, convenient wiring,and can be real-time monitoring environment of network coverage area.To manually adjust the inappropriate environment.it improves the flexibility and security control system of the farms, and improve the economic benefits of farms.

\section{References}

[1] Lv Jiuyi,the research of monitoring system for micro-irrigation based on wireless sensor networks,master degree theses of master of Panyang buiding university, 2011

[2] Deng Ying, Zhang li, Liu you-yuan , intelligent building security system based on wireless sensor network (WSN) research [J], China shipping, 2007. (5) : 133-134

[3] Chai Shujuan, Zhao jianping, the water quality monitoring system based on wireless sensor network research [J], shandong, qufu normal university, 2010 (36) 75-77.

[4] Yue Zhenhua, based on the research and development of the wireless sensor network (WSN), master degree theses of master of jilin university, 2010

[5] design of the wireless sensor network system based on CC2430, 2010.11 electronics world.

[6] Ding en-jie , Meng Xiang, Li xiao,Dai ji. Hydraulic support based on wireless sensor network (WSN) underground pressure monitoring system

[7] Gong Daoli, environment monitoring system based on wireless sensor network (WSN), master degree theses of master of China university of geosciences, 2011 